\title{
ANÁLISE DA NOTIFICAÇÃO DE EVENTOS ADVERSOS EM HOSPITAIS ACREDITADOS*
}

\author{
Tatiana Paula Miguelaci Ferezin ${ }^{1}$, Daniele Ramos², Graziela Caldana ${ }^{2}$, Carmen Silvia Gabriel ${ }^{3}$, Andrea \\ Bernardes $^{3}$
}

RESUMO: Objetivou-se analisar a notificação de eventos adversos em hospitais acreditados do interior de São Paulo, sob a perspectiva da equipe de enfermagem. Estudo descritivo, tipo survey, transversal, com abordagem quantitativa, realizado em três hospitais acreditados do interior do estado de São Paulo, com amostra de 61 enfermeiros e 250 técnicos e auxiliares de enfermagem. A coleta de dados ocorreu entre novembro de 2014 e abril de 2015. Demonstrou-se que $126(75,4 \%)$ participantes possuíam conhecimento para realizar notificação de eventos adversos, mas apenas $109(65,3 \%)$ relataram ter autorização para realizá-las, sendo os enfermeiros apontados pelos participantes como responsáveis por esta ação. Do total de participantes, $76(45,5 \%)$ afirmaram que a notificação dos eventos gera medidas punitivas para os profissionais envolvidos. Apesar disso, $62(37,1 \%)$ não destacaram medo de punição como fator dificultador. A instituição deve priorizar o aprendizado e a cultura de segurança, focada na melhoria da qualidade do atendimento.

DESCRITORES: Qualidade da assistência à saúde; Avaliação de serviços de saúde; Segurança do paciente; Acreditação hospitalar; Enfermagem.

\section{ANALYSIS OF ADVERSE EVENT REPORTING AT ACCREDITED HOSPITALS}

ABSTRACT: The objective was to analyze the reporting of adverse events at accredited hospitals in the interior of the state of São Paulo from the nursing team's perspective. Descriptive and cross-sectional survey with a quantitative approach, undertaken at three accredited hospitals in the interior of the state of São Paulo, with a sample of 61 nurses and 250 nursing technicians and auxiliary nurses. The data were collected between November 2014 and April 2015. It was demonstrated that 126 (75.4\%) participants were knowledgeable to report adverse events, but only 109 (65.3\%) reported being authorized to do so. The participants appointed the nurses as responsible for this action. In the total group of participants, 76 (45.5\%) affirmed that the event reporting entails punitive measures for the professionals involved. Nevertheless, $62(37,1 \%)$ did not highlight fear of punishment as a hindering factor. The institution should prioritize the learning and the safety culture, focused on improving the quality of care.

DESCRIPTORS: Quality of health care; Health services evaluation; Patient safety; Hospital accreditation; Nursing.

\section{ANÁLISIS DE LA NOTIFICACIÓN DE EVENTOS ADVERSOS EN HOSPITALES ACREDITADOS}

RESUMEN: La finalidad fue analizar la notificación de eventos adversos en hospitales acreditados del interior del estado de São Paulo, bajo la perspectiva del equipo de enfermería. Estudio descriptivo, tipo survey, transversal, con aproximación cuantitativa, desarrollado en tres hospitales acreditados del interior del estado de São Paulo, con muestra de 61 enfermeros y 250 técnicos y auxiliares de enfermería. Los datos fueron recolectados entre noviembre del 2014 y abril del 2015. Se demostró que 126 (75,4\%) participantes poseían conocimiento para notificar eventos adversos, pero solamente 109 (65,3\%) relataron contar con la autorización para efectuarlas, siendo los enfermeros apuntados por los participantes como responsables por esta acción. Del total de participantes, $76(45,5 \%)$ afirmaron que la notificación de los eventos genera medidas punitivas para los profesionales involucrados. Aunque así, $62(37,1 \%)$ no destacaron medo de punición como factor dificultador. La institución debe priorizar el aprendizaje y la cultura de seguridad, con foco en la mejora de la calidad de la atención.

DESCRIPTORES: Calidad de la atención de salud; Evaluación de servicios de salud; Seguridad del paciente; Acreditación de hospitales; Enfermería.

\footnotetext{
*Artigo extraído da dissertação intitulada: "Avaliação da notificação de eventos adversos em hospitais acreditados. Escola de Enfermagem de Ribeirão Preto". Universidade de São Paulo, 2015.
}

${ }^{1}$ Enfermeira. Mestre em Ciências da Saúde. Enfermeira na empresa Sermed Saúde LTDA. Ribeirão Preto, SP, Brasil.

${ }^{2}$ Enfermeira. Doutoranda em Ciências da Saúde. Universidade de São Paulo. Ribeirão Preto, SP, Brasil.

${ }^{3}$ Enfermeira. Pós-doutora em Ciências da Saúde. Docente de Enfermagem da Escola de Enfermagem de Ribeirão Preto, Universidade de São Paulo. Ribeirão Preto, SP, Brasil.

Autor Correspondente:

Tatiana Paula Miguelaci Ferezin

Universidade de São Paulo

Av. dos Bandeirantes, 3900 - 14040-902 - Ribeirão Preto, SP, Brasil

tatianamiguelaci@gmail.com
Recebido: $07 / 12 / 2016$

Finalizado: $19 / 04 / 2017$ 


\section{INTRODUÇÃO}

O movimento mundial pela segurança do paciente e a criação de programas que buscam garantir a qualidade da assistência têm contribuído para o crescente interesse pela temática da notificação e prevenção de eventos adversos (EA) nos sistemas de saúde.

Mesmo com avanços, o erro humano tem destaque nos episódios envolvendo riscos para a segurança do paciente e é amplamente divulgado pela imprensa e mídia, gerando preocupações sociais ${ }^{(1)}$. A falta de compreensão sobre o erro gera nos profissionais um sentimento negativo e, somado à cultura punitiva ainda existente, acarreta em omissão por parte deles ${ }^{(1)}$.

Os EA são conhecidos como resultados negativos em saúde, incidentes ou circunstância que têm potencial para causar danos aos pacientes. Esses resultados podem também ser um erro, sendo este definido como a incapacidade de realizar uma ação planejada conforme pretendida ou a aplicação incorreta de um plano. Erro por definição é sempre não intencional, e pode ou não ocasionar um EA. Ao se reduzir os erros, as chances da ocorrência de EA serão minimizadas ${ }^{(2)}$.

Os incidentes podem ser: incidentes sem danos, incidentes com dano (eventos adversos), ou near misses (quase erro), caracterizado quando um incidente poderia atingir o paciente, causando danos ou não, mas foi interceptado antes de chegar a ocorrer ${ }^{(2)}$.

No Brasil, a notificação de EA pelos serviços de saúde tornou-se obrigatória a partir de 2013, tendo em vista a criação do Programa Nacional de Segurança do Paciente (PNSP) ${ }^{(3)}$. Destaca-se, no cenário nacional, o sistema informatizado NOTIVISA, da Agência Nacional de Vigilância Sanitária (Anvisa), utilizado para o registro de problemas relacionados ao uso de tecnologias e de processos assistenciais, por meio do monitoramento da ocorrência de queixas técnicas de medicamentos e produtos para a saúde, incidentes e EA assistenciais ${ }^{(4)}$.

Vale destacar a necessidade de se ampliar esforços para construção de sistemas de notificação robustos e eficientes, capacitação de profissionais qualificados, atentos e capazes de identificar possíveis erros e eventos, de forma a contribuir para um ambiente mais seguro e livre de danos. A responsabilidade pela segurança no ambiente de trabalho deve ser compartilhada entre as instituições educacionais e as instituições de saúde, já que a temática perpassa ambas as áreas e ainda carece de ações multidisciplinares para que haja mudança no cenário.

Apesar da relevância do tema, ainda existem lacunas de conhecimento nesse campo, principalmente em países menos desenvolvidos. A falta de recursos, infraestrutura e informatização limita a capacidade desses países em coletar e sistematizar informações, desenvolver projetos de pesquisas no campo e, além disso, as notificações e taxas de ocorrência de EA podem estar subestimadas, de modo a não retratar a real extensão e os verdadeiros danos causados ${ }^{(5-6)}$.

No que tange à notificação de EA, há que se ressaltar o papel do enfermeiro no sentido de melhorar o processo de identificação de riscos de eventos e por ser ele o profissional responsável técnico pelos outros membros da equipe de enfermagem. Assim, deve exercer importante função de educador, promovendo a mobilização no sentido de reduzir os EA.

A enfermagem é a profissão que permanece mais tempo com o paciente, estando apta para promover estratégias para prevenção de eventos e promoção da segurança do paciente ${ }^{(7)}$.

É importante estabelecer estratégias nacionais que incentivem a melhoria dos processos nos serviços de saúde e, consequentemente, a melhoria contínua da saúde da população. Soma-se a essas estratégias a necessidade de adotar um sistema de avaliação da qualidade robusto.

Diante dessa necessidade, as instituições com programas de acreditação apresentam destaque com relação ao tema de qualidade da assistência em saúde, uma vez que devem exercer ações capazes de minimizar os EA em serviços de saúde.

A acreditação é um processo de avaliação e promoção da melhoria da qualidade, realizada de forma voluntária e periódica através de padrões pré-estabelecidos, culminando em transformações de hábitos, valores e comportamentos dos indivíduos dentro das instituições adeptas, o que contribui 
para o cuidado qualificado e seguro em prol da excelência ${ }^{(8-9)}$. Cabe a ressalva de que esse processo também exige que os estabelecimentos de saúde atuem em conformidade com os requisitos técnicos e legais e tenham seu licenciamento validado pela Anvisa.

Diante do exposto, o estudo objetivou analisar a notificação de EA em hospitais acreditados do interior do estado de São Paulo (SP), na perspectiva da equipe de enfermagem.

\section{MÉTODO}

Estudo descritivo, do tipo survey, transversal, com abordagem quantitativa. O estudo foi realizado intencionalmente em três instituições localizadas no interior do estado de SP, as quais possuem certificado de acreditação pela Organização Nacional de Acreditação (ONA). Entende-se que, por utilizarem a ferramenta da acreditação, elas já possuem sistemas formalizados de notificação e análise de EA, sendo consideradas, portanto, cenários adequados para responder aos objetivos desta investigação justificando, assim, a intencionalidade dos cenários.

Foram participantes do estudo os enfermeiros, técnicos e auxiliares de enfermagem de três hospitais $(\mathrm{N}=697)$, de uma cidade do interior do estado SP, acreditados pela ONA, escolhidos intencionalmente por entender-se que por utilizarem a ferramenta da acreditação, necessariamente as instituições já possuem sistemas formalizados de notificação e análise de EA, tendo em vista que a certificadora preconiza essa estratégia em seus manuais.

Foi utilizado como critério de inclusão ser profissional de enfermagem que presta assistência direta ao paciente e, como critério de exclusão, os profissionais de enfermagem com tempo de trabalho inferior a seis meses na instituição.

Para o cálculo amostral, foram utilizadas as seguintes fórmulas: $n=\frac{z_{\alpha / 2}^{2} P(1-P)}{\varepsilon^{2}}$ e $n_{h}=n \frac{N_{h}}{N}$ onde na primeira $\mathrm{P}=$ prevalência do evento, = nível de significância e o = erro tolerável, e na segunda $\mathrm{Nh}=$ total de cada estrato por hospital; $\mathrm{h}=$ estrato por hospital e $\mathrm{N}=$ população. Foi atribuído perda de 0,2 , nível de significância de $5 \%$ e erro relativo de $10 \%$. O programa utilizado pelo estatístico para esta análise foi o $R$ versão 3.0.2. Após o cálculo da amostra, esta foi aleatorizada através do programa Statistical Package for the Social Sciences (SPSS) 16.0.

A instituição A, um hospital geral com atendimento clínico e cirúrgico, possui 94 leitos, sendo acreditado em nível III. A instituição B é um hospital especializado, com 21 leitos, acreditado em nível I. Já a instituição C é um hospital geral com atendimento clínico e cirúrgico, sendo acreditado em nível II. Todos os hospitais são acreditados nos níveis descritos pela ONA.

Para a coleta de dados, realizada entre os meses de novembro de 2014 e abril de 2015, foi utilizado questionário elaborado pelos pesquisadores, constituído por dezoito questões fechadas e duas questões abertas relacionadas à notificação de eventos adversos, além de dados de caracterização.

Parte das questões fechadas foi construída adotando-se escala de concordância, que consiste em várias afirmações declaratórias (itens) que expressam um ponto de vista sobre um assunto. Os participantes são solicitados a indicar o grau em que concordam ou discordam da opinião expressa pela afirmação ${ }^{(10)}$.

Para a realização da validade de face e conteúdo do instrumento, foi utilizado método baseado no julgamento por meio da seleção de um grupo de três juízes que atuam e/ou que sejam referência no ensino e/ou na pesquisa sobre segurança do paciente e enfermagem.

Foram escolhidos três juízes para evitar questionamentos dúbios e eliminar o risco de empate na avaliação, que receberam o questionário, analisaram e propuseram ajustes, inclusão e exclusão de termos.

O instrumento possui dimensões relacionadas à melhoria de segurança do paciente, características sobre os eventos notificados, ações punitivas ou não punitivas e dificuldades e potencialidades para 
notificação.

A análise dos dados foi realizada de forma descritiva através do programa SPSS 16.0 para construir a base de dados, gerenciá-los, tratá-los e executar as análises estatísticas descritivas e o cálculo da confiabilidade. Para a elaboração dos gráficos, foi utilizado o Microsoft Excel ${ }^{\circledast}$.

Cada item do questionário foi avaliado segundo o percentual de respostas positivas, obtido por meio do cálculo da combinação das duas categorias mais altas de resposta (concordo totalmente e concordo), sendo as questões referentes às atividades de melhoria de segurança do paciente e fatores positivos mediante a notificação de eventos adversos. Já para o item de número 12, no qual é questionada a aplicação de punição após a notificação de eventos adversos pelo profissional, o percentual de respostas positivas foi obtido por meio do cálculo da combinação das duas categorias mais baixas de resposta (discordo e discordo totalmente).

O estudo foi avaliado e aprovado pelo Comitê de Ética em pesquisa da Escola de Enfermagem de Ribeirão Preto, Universidade de São Paulo, com parecer n 704.078.

\section{RESULTADOS}

A distribuição dos participantes segundo categorias profissionais por instituição está descrita na Tabela 1.

Tabela 1 - Número de participantes segundo categorias profissionais por instituição. Ribeirão Preto, SP, Brasil, 2015

\begin{tabular}{lcccc} 
Instituição & \multicolumn{2}{c}{ Enfermeiros } & \multicolumn{2}{c}{ Técnicos/Auxiliares enfermagem } \\
\cline { 2 - 5 } & $\mathbf{N}^{*}$ & $\mathbf{N}$ & $\mathbf{N}^{*}$ & $\mathbf{n}$ \\
\hline A & 92 & 325 & 41 & 145 \\
\hline B & 11 & 48 & 5 & 21 \\
\hline C & 34 & 187 & 15 & 84 \\
\hline Total & 137 & 560 & 61 & 250
\end{tabular}

* Dados fornecidos pelo Recursos Humanos das instituições participantes e cálculo amostral.

A caracterização dos participantes está apresentada na Tabela 2. A idade dos participantes se encontrou predominantemente na faixa de 20 a 40 anos, mínima de 20 anos, máxima de 58, com predominância de profissionais adultos jovens. A maioria dos participantes tem de 1 a 15 anos de trabalho na enfermagem, sendo que técnicos de enfermagem perfizeram um total de $84(50,3 \%)$ participantes. Dos participantes, $96(57,5 \%)$ responderam que trabalham somente na instituição pesquisada.

Tabela 2 - Caracterização dos participantes da pesquisa segundo variáveis sociodemográficas. Ribeirão Preto, SP, Brasil, 2015 (continua)

\begin{tabular}{|c|c|c|c|}
\hline Variáveis sociodemográficas & & $\mathbf{n}$ & $\%$ \\
\hline \multirow[t]{3}{*}{ Função na instituição } & Auxiliar de enfermagem & 50 & 29,9 \\
\hline & Técnico de enfermagem & 84 & 50,3 \\
\hline & Enfermeiro & 33 & 19,8 \\
\hline \multirow[t]{5}{*}{ Tempo de trabalho na enfermagem } & $<1$ ano & 3 & 1,8 \\
\hline & $1-15$ anos & 133 & 79,6 \\
\hline & $16-30$ anos & 24 & 14,4 \\
\hline & $>30$ anos & 1 & 0,6 \\
\hline & Missing & 6 & 3,6 \\
\hline
\end{tabular}




\begin{tabular}{|c|c|c|c|}
\hline \multirow[t]{4}{*}{ Tempo de trabalho na instituição } & $<1$ ano & 39 & 23,4 \\
\hline & $1-15$ anos & 113 & 67,7 \\
\hline & $16-30$ anos & 8 & 4,8 \\
\hline & Missing & 7 & 4,1 \\
\hline
\end{tabular}

$\mathrm{Na}$ análise das questões relacionadas aos EA, verificou-se que $126(75,4 \%)$ participantes referiram possuir conhecimento sobre o sistema de notificação das instituições. Isoladamente, 30 (91\%) dos enfermeiros referiram conhecer o sistema de notificação.

Apesar do destaque para o papel do enfermeiro como principal notificador, houve um número significativo de autorizações para a notificação $(n=109 ; 65,3 \%)$, sendo que o conhecimento do fluxo das notificações foi referido por 22 (67\%) enfermeiros e 79 (47,3\%) técnicos e auxiliares de enfermagem.

Os meios de notificação de eventos relatados foram: a via online em 104 (62,3\%) casos, seguida de impresso específico em 48 (28,7\%) casos, relatório escrito em 30 (18\%) e, ainda, oito (4,8\%) participantes informaram outro meio.

Paradoxalmente, apesar de $126(75,4 \%)$ do total de participantes apontarem a existência de capacitações para realização da notificação, entre esses, 24 (19\%) relataram não conhecer o sistema de notificação. Reitera-se que 27 (81,8\%) enfermeiros concordam que a instituição promove ou promoveu capacitações para utilização do sistema e realização das notificações.

Os dados demonstraram que $102(61,1 \%)$ participantes afirmaram que a notificação dos eventos é anônima e/ou voluntária, esta afirmação foi aplicada em uma mesma questão.

Apesar dos participantes apontarem que o sistema de notificação de EA das instituições em que trabalham é fácil de usar 225 (72,5\%), destes, 21 (17,4\%) apontaram dificuldades com relação ao sistema como principal problema para realização da notificação.

Quanto aos motivos para ausência de notificação, tanto os técnicos e auxiliares de enfermagem quanto os enfermeiros destacaram a falta de tempo, sendo que as respostas revelaram $72(43,1 \%)$ e $17(51,5 \%)$ respectivamente para as categorias. Cabe destacar que, entre os enfermeiros, o medo de punição também foi apontado no item em 13 (21,2\%) casos. Corroborando o item em que nove (27,3\%) enfermeiros relataram haver medidas punitivas aplicadas pelas instituições quando há ocorrência de EA, conforme demonstrado na Tabela 3.

Tabela 3 - Comparação entre enfermeiros e técnicos/auxiliares de enfermagem referente ao entendimento sobre ocorrência de medidas punitivas, retorno ao notificador e discussão sobre causas. Ribeirão Preto, SP, Brasil, 2015

\begin{tabular}{|c|c|c|c|c|}
\hline \multirow[t]{2}{*}{ Respostas } & \multicolumn{2}{|c|}{ Enfermeiros } & \multicolumn{2}{|c|}{$\begin{array}{l}\text { Técnicos/Auxiliares } \\
\text { de enfermagem }\end{array}$} \\
\hline & $\mathbf{n}$ & $\%$ & $\mathbf{n}$ & $\%$ \\
\hline Concordam haver medidas punitivas & 9 & 27,3 & 67 & 50 \\
\hline $\begin{array}{l}\text { Concordam que o notificador e/ou setor tem retorno sobre sua } \\
\text { notificação }\end{array}$ & 18 & 54,5 & 90 & 67,2 \\
\hline $\begin{array}{l}\text { Concordam haver discussão sobre possíveis causas dos eventos } \\
\text { notificados e como evitá-las }\end{array}$ & 22 & 66,7 & 96 & 71,6 \\
\hline
\end{tabular}

Os participantes concordaram que os eventos ocorridos levam a mudanças positivas para segurança $132(79,1 \%)$ e haver discussão de estratégias de prevenção que garantam que estes eventos não voltem a ocorrer $128(76,7 \%)$.

No que tange às ferramentas de análise da notificação dos eventos ocorridos, os enfermeiros 
citaram: comissão de qualidade, espinha de peixe, indicadores, análise de causa raiz e a classificação de risco. Já os técnicos e auxiliares de enfermagem destacaram: capacitações, reuniões, gestão de qualidade, discussões, protocolo, a própria ONA, gráficos, formulário específico/protocolo interno, comissão de infecção hospitalar, ferramenta própria on-line.

A existência de atividades de melhoria para segurança do paciente na instituição foi apontada por $284(91,6 \%)$ participantes, que destacaram entre elas: o uso do checklist de cirurgia segura, pulseira de identificação do paciente, cor de pulseira diferente para paciente alérgico, identificação e uso de grades nos leitos, realização de capacitações, implantação de protocolos, prevenção de úlcera por pressão/ mudança de decúbito, a própria notificação de eventos ocorridos, uso de etiquetas em medicações de alto risco, e educação continuada.

\section{DISCUSSÃO}

A preponderância de técnicos de enfermagem entre os profissionais do estudo corrobora os dados do Conselho Federal de Enfermagem (COFEN) para o panorama nacional, demonstrando que estavam cadastrados em março de 2010, 287.119 (19,8\%) enfermeiros, 625.863 (43,2\%) técnicos e 533.422 (36,8\%) auxiliares de enfermagem, havendo discreto aumento para categoria técnica ${ }^{(11)}$.

Ao considerar a precarização do trabalho da enfermagem no país, resultante de diversos motivos, tais como a desregulamentação, ausência de direitos, jornadas extensas, complexidade das condições de trabalhistas e os baixos salários, apontados como possível causa de vínculos diversos ${ }^{(12-13)}$, a sobrecarga de trabalho é inevitável.

O aumento do risco de mortes em pacientes e a ocorrência de eventos adversos estão intimamente relacionados à sobrecarga de trabalho da equipe de enfermagem, cabendo aos gerentes das equipes a participação na gestão de pessoas para a diminuição da sobrecarga e aumento da segurança do paciente $\mathrm{e}^{(14)}$.

Em relação à segurança do paciente, a notificação dos eventos adversos torna-se um instrumento essencial, uma vez que propicia auxílio à gestão da assistência ${ }^{(15)}$. No presente estudo, percebeu-se que os enfermeiros conhecem o sistema de notificação e são os principais notificadores, entretanto, devem desempenhar melhor seu papel de líder da equipe, apoiando, disseminando e informando ações em prol da notificação bem como os resultados das análises das notificações.

Cabe destacar que o sistema de notificação de deve ser de conhecimento de todos os trabalhadores das equipes assistenciais que atuam na instituição, com destaque para a equipe de enfermagem. A falta de conhecimento acerca do que é, como se identifica e como se notifica o EA em saúde, são dados que podem ser maiores do que os que se tem oficialmente divulgados ${ }^{(16)}$.

Apesar de umavisão fragmentada da equipe de enfermagem acerca da segurança do paciente, a notificação e responsabilidade pelo EA devem ser transversais, sendo que todos os membros devem estar sensibilizados e envolvidos para realizá-la e traçar estratégias para redução de danos e prevenção de novas ocorrências ${ }^{(17-18)}$.

Cabe destacar que, em hospitais acreditados, a segurança do paciente e, por conseguinte, a notificação de EA, é um dos pilares do sistema de qualidade. Através das notificações, além de outros instrumentos, obtém-se informações para evitar e reduzir danos à saúde e para a melhoria no sistema de saúde, tanto na perspectiva do paciente quanto do trabalhador.

Os profissionais têm insegurança ao realizar notificação quando não há conhecimento de seu fluxo, o que corrobora o entendimento que toda a equipe deve conhecer o fluxo e dinâmica dos processos, destacando-se a necessidade da adoção de uma metodologia confiável, apoiada por instrumentos eficazes e que permita alcançar objetivos previamente definidos ${ }^{(19-20)}$.

As instituições devem refletir criticamente sobre o papel que os líderes devem desempenhar, uma vez que suas decisões estratégicas incluem a gestão de pessoas com vistas à capacitação das equipes para melhoria da segurança ${ }^{(7)}$. Portanto, políticas de capacitação sobre o sistema e o fluxo das notificações devem ser de escopo institucional e não uma iniciativa restrita a uma categoria ou setor. 
O processo para prevenção do evento advém de mudanças culturais e do aumento na notificação de ocorrências, sendo essencial que a liderança e a gestão acreditem em uma cultura não punitiva e abordagem sistêmica do ocorrido, acolhendo e fornecendo subsídios ao profissional envolvido para que as falhas não aconteçam novamente ${ }^{(17)}$.

No intuito de proteção dos profissionais envolvidos no processo de notificação dos EA, frente a uma cultura ainda punitiva, para contribuir para a identificação das situações de risco e seu gerenciamento, as notificações devem ser preferencialmente anônimas, confidenciais e não serem utilizadas como instrumento de acusação de profissionais ${ }^{(21)}$.

Apesar da discussão sobre a aplicação de medidas punitivas aos profissionais mediante o evento ocorrido, verificou-se que o "medo da punição" não foi apontado como o principal fator dificultador para notificação pelos técnicos e auxiliares de enfermagem.

Nesse contexto, os enfermeiros e chefias das instituições devem ser o apoio para os profissionais que estão na linha de frente da assistência, proporcionando aprendizado e segurança para realização de suas atividades e incentivando a notificação por meio de condutas menos punitivas e mais educativas.

Em relação à postura positiva frente ao EA, os resultados deste estudo corroboraram com estudo brasileiro semelhante, em que $89,8 \%$ enfermeiros coordenadores concordaram que a discussão sobre os eventos tem levado a mudanças positivas ${ }^{(7)}$.

O retorno sobre a notificação para o profissional é capaz de estabelecer um canal de comunicação que possibilita à instituição fornecer um parecer sobre sua conduta, orientação, informações sobre a estratégia para a resolução do problema e necessidade de reavaliação(15). As discussões provenientes dos resultados de análises de EA podem servir como base para a tomada de decisão e para planejamento da segurança do paciente a partir da análise, monitoramento, minimização e prevenção de incidentes que possam causar $\mathrm{EA}^{(22)}$.

Por toda sua importância, de acordo com PNSP, a linguagem a ser utilizada pelo sistema de notificação de eventos deve ser adaptada e amigável, de forma a promover a participação dos diversos profissionais ${ }^{(3)}$.

A monitorização dos incidentes é importante para a garantia da segurança dos pacientes e depende de esforços para que a identificação deles seja feita antes que causem dano, ou seja, os riscos devem ser identificados a tempo de implementar melhorias que evitem o resultado negativo, e esse processo deve ser contínuo, visto que os riscos são inerentes.

Considera-se como limitação deste estudo a aplicação de questionários com preenchimento apenas pela equipe de enfermagem, tendo em vista que o processo de notificação de EA deve ser uma prática de todos os profissionais que atuem nessas instituições. Além disso, a coleta de dados apenas por meio de questionário, sem observar a prática cotidiana dos profissionais que participaram.

\section{- CONSIDERAÇÕES FINAIS}

O presente estudo contribuiu para avaliar as fragilidades e potencialidades dos sistemas de notificação de EA nas instituições acreditadas investigadas. A maioria dos participantes conhece o sistema de notificação de eventos da instituição, porém, não conhece o fluxo do sistema de notificação utilizado. O sistema de notificação das instituições é anônimo e voluntário, fator considerado importante para evitar subnotificações e para incentivar a equipe a notificar sem medo de punições.

Os participantes caracterizam a falta de tempo como principal fator dificultador na realização das notificações, e a maioria dos pesquisados não concorda que haja medidas punitivas nas instituições.

Observaram-se vários pontos positivos e relevantes com relação à notificação de eventos nos hospitais pesquisados, assim como algumas fragilidades que devem ser analisadas pelos gestores e pelos líderes, proporcionando aprimorar os processos de trabalho, melhorar a qualidade da assistência e a segurança dos cuidados prestados.

Entre as fragilidades, destaca-se a importância de ampliar o conhecimento da equipe de enfermagem 
sobre notificação de EA, motivando a equipe para que todos atuem como participantes ativos nesta atividade.

Faz-se necessário, ainda, maior investimento das instituições de saúde nos sistemas de notificação para que toda equipe de saúde realize e tenha conhecimento da dinâmica do processo de notificação de eventos, independente do grau de gravidade.

\section{REFERÊNCIAS}

1. Duarte SCM, Stipp MAC, da Silva MM, de Oliveira FT. Eventos adversos e segurança na assistência de enfermagem. Rev. bras. enferm. [Internet] 2015;68(1) [acesso em 14 out 2016]. Disponível: http://dx.doi. org/10.1590/0034-7167.2015680120p.

2. World Health Organization. The Conceptual Framework for the International Classification for Patient Safety. [Internet] 2009 [acesso em 20 abr 2014]. Disponível: http://www.who.int/patientsafety/taxonomy/icps_chapter3. pdf.

3. Ministério da Saúde (BR). Gabinete do Ministro. Portaria n. 529, de $1^{\circ}$ de abril de 2013. Institui o Programa Nacional de Segurança do Paciente (PNSP). Diário Oficial da União, Brasília, 02 abr. 2013. Seção 1.

4. Ministério da Saúde (BR). Agência Nacional de Vigilância Sanitária. Resolução da Diretoria Colegiada - RDC n. 36, de 25 de julho de 2013. Institui ações para a segurança do paciente em serviços de saúde e dá outras providências. Diário Oficial da União, Brasília, 26 jul. 2013. Seção 1.

5. Carpenter KB, Duevel MA, Lee PW, Wu AW, Bates DW, Runciman WB, et al. Measures of patient safety in developing and emerging countries: a review of the literature. Qual Saf Health Care. [Internet] 2010;19(1) [acesso em 20 set 2016]. Disponível: http://dx.doi.org/10.1136/qshc.2008.031088.

6. Wilson RM, Michel P, Olsen S, Gibberd RW, Vincent C, El-Assady R, et al. Patient safety in developing countries: retrospective estimation of scale and nature of harm to patients in hospital. BM]. [Internet] 2012;(344) [acesso em 20 set 2016]. Disponível: http://dx.doi.org/10.1136/bmj.e832.

7. Françolin L, Gabriel CS, Bernardes A, de Camargo e Silva AEB, Brito MFP, Machado JP. Gerenciamento da segurança do paciente sob a ótica dos enfermeiros. Rev. esc. enferm. USP. 2015;49(2):277-83.

8. Organização Nacional de Acreditação (ONA). Manual brasileiro de acreditação: organizações prestadoras de serviços de saúde. Brasília: ISQua ONA; 2014.

9. Siman AG, Brito MJM, Carrasco MEL. Participação do enfermeiro gerente no processo de acreditação hospitalar. Rev. Gaúcha Enferm. 2014;35(2):93-9.

10. Polit D F, Beck CT. Nursing research: generating and assessing evidence for nursing practice. $9^{\mathrm{a}}$ ed. Philadelphia: Lippincott Williams \& Wilkins; 2012.

11. Conselho Federal de Enfermagem (COFEN). Comissão de Business Intelligence. Análise de dados dos profissionais de enfermagem existentes nos Conselhos Regionais. Brasília: COFEN; 2011.

12. de Lima AAC, de Lacerda JC, de Melo NA, de Lima SS, Grando LH. Estresse do profissional de enfermagem: revisão de literatura stress of professional nursing: literature review. Rev Saúde-UnG. 2014;8(3-4):73.

13. Montanholi LL, Tavares DMS, de Oliveira GR. Estresse: fatores de risco no trabalho do enfermeiro hospitalar. Rev. bras. enferm. 2006;59(5):661-5.

14. Novaretti MCZ, Santos EV, Quitério LM, Daud-Gallotti RM. Nursing workload and occurrence of incidents and adverse events in ICU patients. Rev. bras. enferm. 2014;67(5):692-9.

15. de Paiva MCMS, Popim RC, Melleiro MM, Tronchim DMR, Lima SAM, Juliani CMCM. The reasons of the nursing staff to notify adverse events. Rev. Latino-Am. Enfermagem. [Internet] 2014;22(5) [acesso em 14 set 2016]. Disponível: http://dx.doi.org/10.1590/0104-1169.3556.2476.

16. da Silva RCL, Cunha JJSA, Moreira CLS. Evento adverso em terapia intensiva: o que sabem os profissionais de 
enfermagem. Rev. pesqui. cuid. fundam. (Online). [Internet] 2011;3(2) [acesso em 21 set 2016]. Disponível: http:// www.seer.unirio.br/index.php/cuidadofundamental/article/view/1393/pdf_384.

17. Fassini $P$, Hahn GV. Riscos à segurança do paciente em unidade de internação hospitalar: concepções da equipe de enfermagem. Rev Enferm UFSM. 20122(2):290-9.

18. Bohomol E, Tartali JA. Eventos adversos em pacientes cirúrgicos: conhecimento dos profissionais de enfermagem. Acta paul. enferm. [Internet] 2013;26(4) [acesso em 15 jul 2014]. Disponível: http://dx.doi.org/10.1590/ S0103-21002013000400012.

19. Gifford ML, Anderson JE. Barriers and motivating factors in reporting incidents of assault in mental healthcare. J Am Psychiatr Nurses Assoc. 2010;16(5):288-98.

20. Ausserhofer D, Schubert M, Engberg S, Blegen M, Geest S, Schwendimann R. Nurse-reported patient safety climate in Swiss hospitals: a descriptive-explorative substudy of the Swiss RN4CAST study. Swiss Med Wkly. [Internet] 2012;(142) [acesso em 12 ago 2016]. Disponível: http://dx.doi.org/10.4414/smw.2012.13501.

21. Hwang JI, Lee SI, Park HA. Barriers to the Operation of Patient Safety Incident Reporting Systems in Korean General Hospitals. Healthc Inform Res. [Internet] 2012;18(4) [acesso em 28 mai 2015]. Disponível: http://dx.doi. org/10.4258/hir.2012.18.4.279.

22. Silva SC. Qualidade nos serviços de saúde e a segurança do ambiente. In: Harada MJCS, Pedreira MLG. Gestão em Enfermagem: ferramenta para prática segura. São Caetano do Sul: Yendis; 2011. p. 305-14. 